\title{
Nasopharyngeal carcinoma treated with bevacizumab combined with paclitaxel liposome plus cisplatin: a case report and literature review
}

This article was published in the following Dove Press journal:

OncoTargets and Therapy

20 December 2016

Number of times this article has been viewed

\author{
Wan He, ${ }^{1, *}$ Chang Zou, ${ }^{2, *}$ \\ Zhongkai Tian,' Wenyong \\ Tan, ' Weixi Shen, ${ }^{3}$ Jinghua \\ Chen,' Liping Liu, ${ }^{4}$ \\ Ruilian Xu' \\ 'Department of Oncology, The Second \\ Clinical Medical College, Shenzhen \\ People's Hospital, Jinan University, \\ Shenzhen, China; ${ }^{2}$ Clinical Medical \\ Research Center, The Second Clinical \\ Medical College, Shenzhen People's \\ Hospital, Jinan University, Shenzhen, \\ China; ${ }^{3}$ Department of Oncology, \\ Shenzhen Hospital of Southern \\ Medical University, Shenzhen, China; \\ ${ }^{4}$ Department of Hepatobiliary and \\ Pancreatic Surgery, The Second \\ Clinical Medical College, Shenzhen \\ People's Hospital, Jinan University, \\ Shenzhen, China \\ *These authors contributed equally \\ to this work
}

Correspondence: Liping Liu Department of Hepatobiliary and Pancreatic Surgery, The Second Clinical Medical College, Shenzhen People's Hospital, Jinan University, Room II I 2I/F, Surgical Building, I0I7 Dongmen North Road, Luohu, Shenzhen, Guangdong, China

Tel +86 I375I037I56

Email94025769@qq.com

Ruilian Xu

Department of Oncology, The Second Clinical Medical College, Shenzhen People's Hospital, Jinan University, Room 329, 3/F, Block 2, 1017 Dongmen North Road, Luohu, Shenzhen, Guangdong,

China

Tel +86 I392388 9I23

Email xuruilian@126.com

\begin{abstract}
Patients with stage IV nasopharyngeal carcinoma (NPC) have a poor prognosis, even with effective chemotherapy. Target agents combined with chemotherapy may improve NPC patients' outcome. The case of a patient with NPC, who was treated by adding bevacizumab to chemotherapy after disease progression using first-line chemotherapy, is reported. Recently published literature about effects of combining bevacizumab with standard chemotherapy in NPC cell lines or patients are also reviewed and discussed. Consistent with a few preclinical trials and Phase II clinical trials, bevacizumab may reverse the drug resistance to chemotherapy, and its toxic side effects are well tolerated.
\end{abstract}

Keywords: nasopharyngeal carcinoma, bevacizumab, drug resistance, combination therapy

\section{Introduction}

Early detection of nasopharyngeal carcinoma (NPC) is difficult. In patients with systemic metastases, the overall survival rates at 5 years were $28 \%-61 \%$ in NPC stage IV. ${ }^{1}$ Target agents combined with chemotherapy may enhance tumor response rate and extend median overall survival. Combination therapy involving target agents against epidermal growth factor receptor (EGFR) such as cetuximab ${ }^{2}$ or nimotuzumab with radiotherapy has been documented. Besides, multiple therapies, including antiangiogenic agents such as bevacizumab ${ }^{3}$ or endostatin, the EGFR tyrosine kinase inhibitor gefitinib, ${ }^{4} \mathrm{COX}-2$ inhibitors, and the anti-HER-2 agent trastuzumab in combination with radiotherapy or chemotherapy against advanced NPC, have been reported in some small studies. In this report, the details of one patient with NPC who was treated with bevacizumab plus chemotherapy is reported and recent literature is reviewed.

\section{Case report}

The patient was a 39-year-old man without any other associated disease who presented with bilateral neck mass for 1 month. Core needle biopsy of the right neck mass revealed lymph node with metastatic carcinoma. Positron emission tomography/computed tomography (PET/CT) scan demonstrated nodules in the left nasopharynx, suggesting malignant neoplasm; metastasis in the left parotid, liver, many vertebra, and lymph nodes was also observed. Nasopharyngoscopy was performed and subsequent histology showed a nonkeratinizing NPC. Thereafter, the patient received three cycles of palliative chemotherapy with paclitaxel liposome $\left(175 \mathrm{mg} / \mathrm{m}^{2}\right.$, day 1$)$, cisplatin $\left(15 \mathrm{mg} / \mathrm{m}^{2}\right.$, days $1-5)$, and 5-fluorouracil (500 mg/ $\mathrm{m}^{2}$, days $\left.1-5\right)$. With chemotherapy, the bilateral neck mass had been shrinking remarkably and the pain in the vertebra also lessened. 
Before the fourth cycle of chemotherapy was delivered, the patient's pain relapsed, appetite decreased, and fatigue and weight loss were observed. The second PET/CT scan (June $18,2013)$ revealed that the nasopharyngeal mass and lymph node metastases were reduced in size, whereas liver and bone metastases had increased in size and number (Figure 1), indicating progressive disease. Bevacizumab (7.5 mg/kg, day 1) was combined with the former chemotherapy for another three cycles. With the addition of bevacizumab to chemotherapy, pain relieved, appetite improved and weight maintained for the patient. The third PET/CT scan (September 2, 2013) demonstrated stable bone metastases and disease reduction in other metastatic sites, suggesting stable disease (SD) (Figure 2). The patient wanted to receive more active therapy to achieve a better outcome than SD. Therefore, three cycles of gemcitabine $\left(1,000 \mathrm{mg} / \mathrm{m}^{2}\right.$, days 1 and 8$)$ and nedaplatin $\left(90 \mathrm{mg} / \mathrm{m}^{2}\right.$, day 1$)$ combined with bevacizumab $(7.5 \mathrm{mg} / \mathrm{kg}$, day 1) were given. Over time, the patient needed analgesics once more to lessen pain and he also lost his appetite. Then, chemotherapy was stopped because of liver dysfunction (serum glutamic-pyruvic transaminase: $78 \mathrm{U} / \mathrm{L}$, serum glutamic-oxal(o)acetic transaminase: $162 \mathrm{U} / \mathrm{L})$. With protective treatment, glutamic-pyruvic transaminase and glutamicoxal(o)acetic transaminase were down into the normal range.

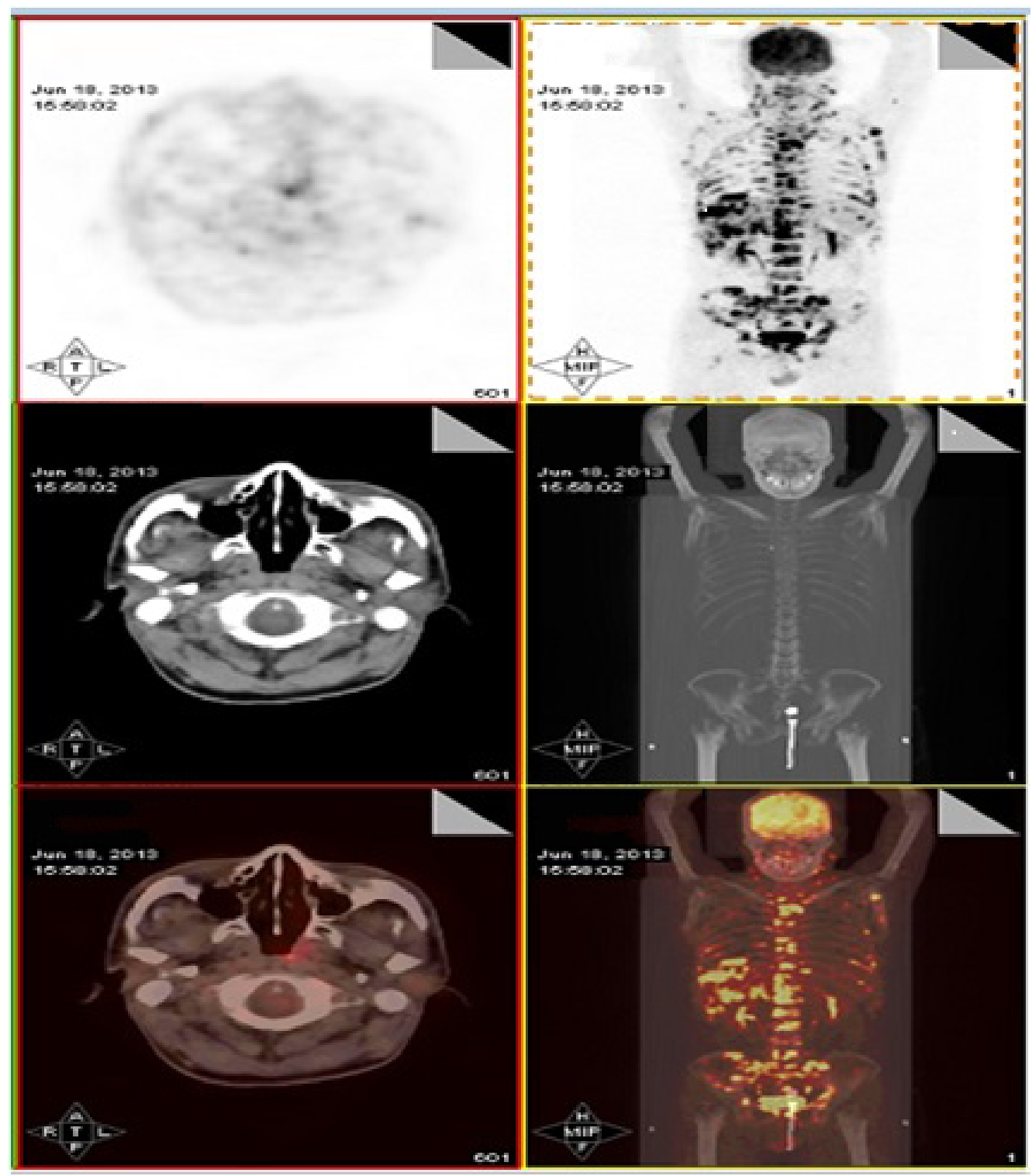

Figure I PET/CT (June 18, 20I3) showing disease extent after first-line chemotherapy.

Notes: Compared with PET/CT images before first-line chemotherapy, this scan revealed that the nasopharyngeal mass and lymph node metastases were reduced in size, whereas liver and bone metastases had increased in size and number, indicating progressive disease.

Abbreviations: CT, computed tomography; PET, positron emission tomography. 


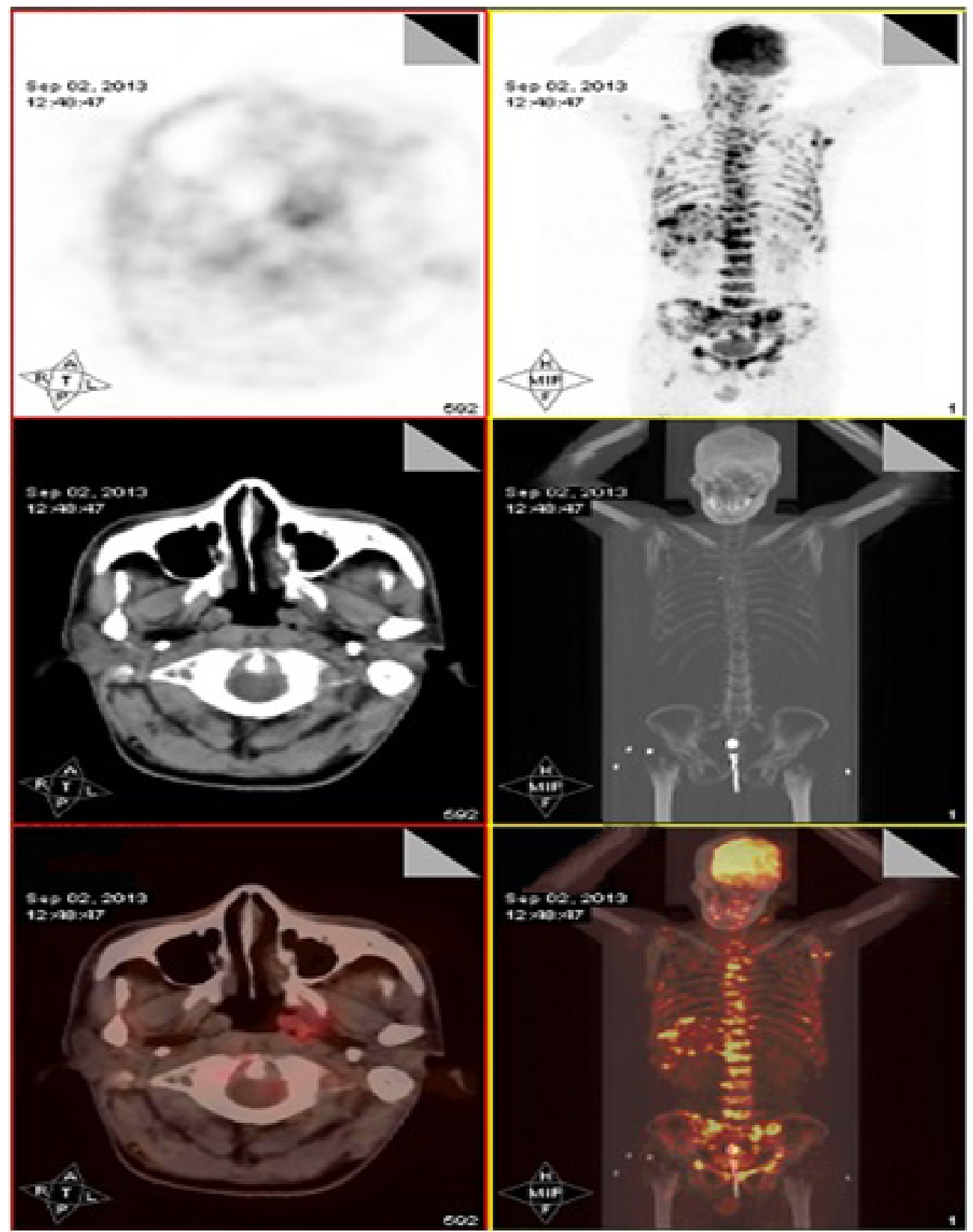

Figure 2 PET/CT (September 2, 20I3) displaying relatively favorable tumor burden with bevacizumab combined with the agents already being administered.

Notes: Although bone metastases remained stable, other metastatic sites including liver metastases were reduced in size and number after addition of bevacizumab to chemotherapy.

Abbreviations: $\mathrm{CT}$, computed tomography; PET, positron emission tomography.

The last chemotherapy was undertaken in December, 2013. The PET/CT was applied again for assessing the response to the above regimen in February 8, 2014 (Figure 3). To control liver metastasis, third-line chemotherapy with gimeracil and oteracil potassium capsules $(60 \mathrm{mg}$, twice a day, days 1-14) was given to the patient; following this, his liver function became abnormal and so he was given the best supportive care. Two months later, the patient died from liver dysfunction and myelosuppression.

\section{Discussion}

NPC, also known as Guangdong carcinoma, has 80\%-90\% 5 -year survival at the early stage. However, its advanced stage has poor prognosis with $61 \%-66 \% 5$-year survival. ${ }^{5,6}$ Multimodality therapy based on chemotherapy is the mainstay for advanced NPC. Recently, EGFR- and vascular endothelial growth factor (VEGF)-targeted therapies have been clinically tested in NPC patients. These target agents involve the anti-EGFR antibody cetuximab ${ }^{2}$ and the anti-VEGF antibody 


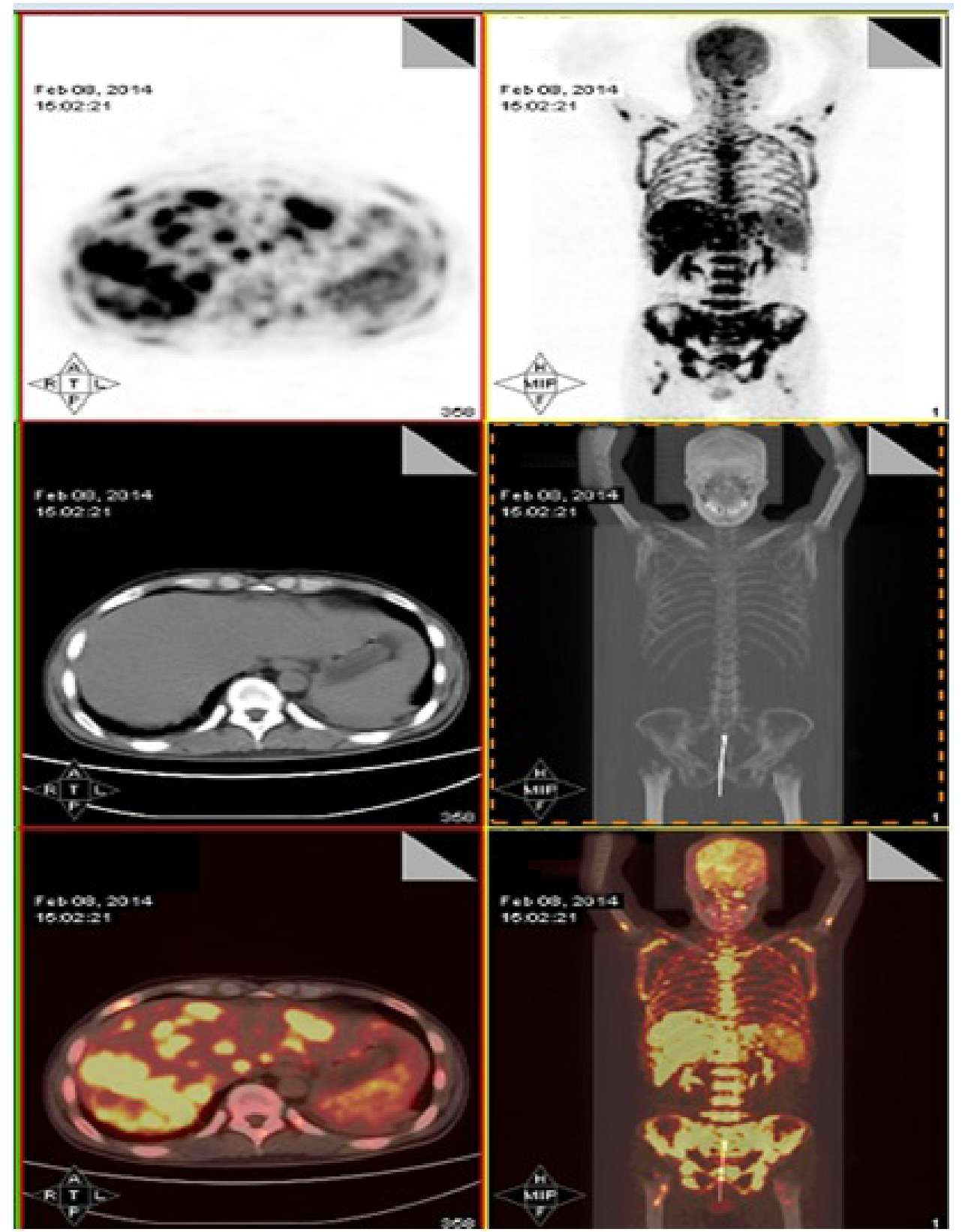

Figure 3 PET/CT (February 8, 20I4) showing significantly progressive disease after changed regimen and discontinuation of treatment because of liver dysfunction. Notes: Liver, bone, and lymph node metastases were increased in size and number. Abbreviations: $\mathrm{CT}$, computed tomography; PET, positron emission tomography.

bevacizumab. ${ }^{3}$ In terms of the role of bevacizumab in NPC, the combination of bevacizumab and chemotherapy in NPC patients has been reported in few studies till date. A few preclinical trials and Phase II clinical trials demonstrated that high expression of VEGF predicted poor prognosis and that anti-VEGF antibody inhibited tumor angiogenesis, promoted tumor cell apoptosis, and reversed resistance to chemotherapy when combined with chemotherapy drugs. ${ }^{3,6,7}$

The VEGF family has seven members: VEGF-A, VEGF-B, VEGF-C, VEGF-D, VEGF-E, placental growth factor PGF, and snake venom VEGF. The protein expression of VEGF is significantly increased in NPC tissue as compared to benign tumors of nasopharyngeal region. The expression of VEGF and the microvessel density are associated with the metastatic potency of NPC tissue and the prognosis of patients with NPC. ${ }^{8}$

VEGF is overexpressed in two-thirds of NPC patients and is associated with lymph node metastasis, recurrence, and reduced overall survival. VEGF contributes to lymph node metastasis by inducing angiogenesis. ${ }^{6} \mathrm{~A}$ study explored 
whether the differential expression of plasma cytokines and angiogenic factors before and after treatment could be prognostic biomarkers for patients with head and neck cancer. The results showed that $7 \%$ of the patients had NPC. During the 37-month follow-up, more patients with decreased level of VEGF were in disease-free survival than those with stable VEGF level. ${ }^{9}$

Beacizumab is a restructured humanized monoclonal antibody that can specifically bind and block VEGF, thus inhibiting tumor angiogenesis. Fujita et $\mathrm{al}^{10}$ reported that bevacizumab plus paclitaxel can significantly inhibit the growth of head and neck carcinoma (including NPC) transplanted tumor, can induce increased density of blood vessels within atrophic tumor cells, and can also induce apoptosis. Combination of bevacizumab with the adenovirus thymidine kinase/ganciclovir suicide gene system was shown to synergistically inhibit the growth of NPC CNE1 cell xenografts, whereas bevacizumab alone had no direct effect on CNE1 cells in vitro. ${ }^{11}$ Also, bevacizumab was able to significantly promote the apoptosis of cisplatin (DDP)-resistant NPC cell lines and restore their sensitivity to DDP, ${ }^{7}$ as was seen in our patient.

Metastasis is the main reason for treatment failure of locally advanced NPC, and patients with high expression of VEGF are more likely to show recurrence and metastasis and have short survival. ${ }^{6,12}$ Recently, a Phase II clinical trial (RTOG0615) showed that the addition of bevacizumab to chemoradiation and subsequent adjuvant chemotherapy can prolong disease-free survival, and side effects were manageable, with only slight bleeding events of grades $1-2 .^{3}$

A small sample report from Nanfang Hospital Cancer Center used gemcitabine combined with bevacizumab to treat patients with advanced refractory NPC: bevacizumab at $3-5 \mathrm{mg} / \mathrm{kg}$, days 1 and 15 ; gemcitabine at $1,000 \mathrm{mg} / \mathrm{m}^{2}$, days 2 and 16; with 28 day repeats. The results showed that 9/10 patients achieved partial response in a shorter time, with four cases maintaining partial response status for more than 10 months. The toxicity was manageable. ${ }^{13}$ In this report, the patient was treated with bevacizumab combined with chemotherapy, which reversed the resistance to chemotherapy, maintained SD, and the progression-free survival was 7 months. The aforementioned preliminary data need to be verified in prospective studies. Professor Li Zhang from Sun Yat-Sen University Cancer Center announced a multicenter randomized Phase II clinical trial to test paclitaxel + carboplatin with or without bevacizumab for the treatment of advanced NPC at the 2014 ASCO meeting. The enrollment of patients began in March 2014 and will last for 2 years. ${ }^{14}$
This clinical trial will help to confirm the effect and safety of adding bevacizumab to paclitaxel and carboplatin for the treatment of advanced NPC patients.

\section{Conclusion}

Prognosis of metastatic NPC remains poor with an 11- to 22-month median overall survival. The role of chemotherapy with target agents in metastasis is well documented and is the mainly palliative treatment. Different from the role of the anti-EGFR antibody cetuximab in treating advanced NPC, addition of the anti-VEGF antibody bevacizumab to chemotherapy in NPC patients warrants further study in both preclinical and clinical settings.

Our results showed preliminary evidence of the activity of bevacizumab in reversing resistance to palliative chemotherapy with paclitaxel liposome, cisplatin, and 5-fluorouracil in a metastatic NPC patient who achieved SD. This information may be useful in the design of combination therapy for advanced NPC. The combination of bevacizumab and chemotherapy is being tested in an ongoing multicenter randomized Phase II clinical trial and will need to be further confirmed.

\section{Acknowledgments}

The study was approved by the Clinical Research Ethics Committee of Shenzhen People's Hospital, China. Written consent was obtained from the patient and his relatives for publication of the case report.

This work was supported by The Health and Family Planning Commission of Shenzhen Municipality (201401013), The Scientific and Technology Foundation of Guangdong Province (2015B090904007), The Natural Science Foundation of Guangdong Province (2015A030313829), and the Science and Technology Planning Project of Guangdong Province (2015B090904007).

\section{Disclosure}

The authors report no conflicts of interest in this work.

\section{References}

1. Zhang L, Chen QY, Liu H, Tang LQ, Mai HQ. Emerging treatment options for nasopharyngeal carcinoma. Drug Des Devel Ther. 2013;7:37-52.

2. Chan AT, Hsu MM, Goh BC, et al. Multicenter, Phase III study of cetuximab in combination with carboplatin in patients with recurrent or metastatic nasopharyngeal carcinoma. J Clin Oncol. 2005;23(15): 3568-3576.

3. Lee NY, Zhang Q, Pfister DG, et al. Addition of bevacizumab to standard chemoradiation for locoregionally advanced nasopharyngeal carcinoma (RTOG 0615): a Phase II multi-institutional trial. Lancet Oncol. 2012;13(2):172-180.

4. Chua DT, Wei WI, Wong MP, Sham JS, Nicholls J, Au GK. Phase II study of gefitinib for the treatment of recurrent and metastatic nasopharyngeal carcinoma. Head Neck. 2008;30(7):863-867. 
5. Leung TW, Tung SY, Sze WK, et al. Treatment results of 1070 patients with nasopharyngeal carcinoma: an analysis of survival and failure patterns. Head Neck. 2005;27(7):555-565.

6. Wakisaka N, Wen QH, Yoshizaki T, et al. Association of vascular endothelial growth factor expression with angiogenesis and lymph node metastasis in nasopharyngeal carcinoma. Laryngoscope. 1999; 109(5):810-814.

7. Sun T, Xu J. Effect of bevacizumab on apoptosis of drug-resistant nasopharyngeal carcinoma cells. Med J Chinese Peoples Liberat Army. 2008;33: 1438-1440.

8. Chua DT, Sham JS, Wei WI, Ho WK, Au GK. The predictive value of the 1997 American Joint Committee on Cancer stage classification in determining failure patterns in nasopharyngeal carcinoma. Cancer. 2001;92(11): 2845-2855.

9. Druzgal CH, Chen Z, Yeh NT, et al. A pilot study of longitudinal serum cytokine and angiogenesis factor levels as markers of therapeutic response and survival in patients with head and neck squamous cell carcinoma. Head Neck. 2005;27(9):771-784.
10. Fujita K, Sano D, Kimura M, et al. Anti-tumor effects of bevacizumab in combination with paclitaxel on head and neck 6 qlJlmUS cell carcinoma. Oncol Rep. 2007;18(18):47-51.

11. Sha D, He YJ, Wang WB, Han JQ. [Anti-nasopharyngeal carcinoma effect in vivo and in vitro of Avastin and adenovirus-thymidine kinase suicide gene]. Zhonghua Er Bi Yan Hou Tou Jing Wai Ke Za Zhi. 2007;42(7): 526-532. Chinese.

12. Huang GW, Masanori S, Lin JE, et al. The relationship between microvessel density, the expression of vascular endothelial growth factor (VEGF), and the extension of nasopharyngeal carcinoma. Laryngoscope. 2000;110(12):2066-2069.

13. Luo RC, Kang SJ. Experimental and clinical research progress in biotherapy for nasopharyngeal. J Oncol. 2008;14:18-21.

14. Zhang L, Huang Y, Qin T, et al. Multicenter, randomized, controlled, open-label study of bevacizumab combined with carboplatin and paclitaxel versus carboplatin and paclitaxel in patients with metastatic nasopharyngeal carcinoma. J Clin Oncol. 2014;32:5s(Suppl; abstr TPS6100).
OncoTargets and Therapy

\section{Publish your work in this journal}

OncoTargets and Therapy is an international, peer-reviewed, open access journal focusing on the pathological basis of all cancers, potential targets for therapy and treatment protocols employed to improve the management of cancer patients. The journal also focuses on the impact of management programs and new therapeutic agents and protocols on

\section{Dovepress}

patient perspectives such as quality of life, adherence and satisfaction The manuscript management system is completely online and includes a very quick and fair peer-review system, which is all easy to use. Visit http://www.dovepress.com/testimonials.php to read real quotes from published authors. 